\title{
Split vortices in optically coupled Bose-Einstein condensates
}

\author{
Juan J. García-Ripoll and Víctor M. Pérez-García \\ Departamento de Matemáticas, Escuela Técnica Superior de Ingenieros Industriales, \\ Universidad de Castilla-La Mancha 13071 Ciudad Real, Spain \\ Fernando Sols \\ Departamento de Física Teórica de la Materia Condensada, \\ Universidad Autónoma de Madrid,Cantoblanco, Spain
}

(Dated: November 9, 2018)

\begin{abstract}
We study a rotating two-component Bose-Einstein condensate in which an optically induced Josephson coupling allows for population transfer between the two species. In a regime where separation of species is favored, the ground state of the rotating system displays domain walls with velocity fields normal to them. Such a configuration looks like a vortex split into two halves, with atoms circulating around the vortex and changing their internal state as they cross the domain wall.
\end{abstract}

PACS numbers: 03.75. Fi, 67.57.Fg, 67.90.+z

Vortex formation is generally viewed as an unequivocal signature of superfluid motion in atomic Bose-Einstein condensates. Both in one- and two-component systems, a number of vortex-like structures have been created and observed [1, 2n that confirm theoretical predictions

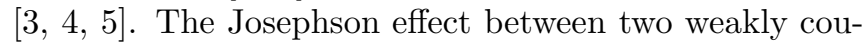
pled condensates is another paradigm of superfluid transport that so far has received less attention [6, 7, 8]. In this article, we present a combined study of these two fundamental properties of superfluid systems: vortex and Josephson dynamics. We study the ground state properties of a rotating double condensate system in which the combined role of vortex formation and optical coupling between two different hyperfine states gives rise to a rich physical behavior.

A crucial consequence of the internal Josephson coupling is the generation of an effective attraction between both atomic species due to the energy that atoms gain by choosing a symmetric mixture of the coupled internal states. Therefore, the most interesting physics is reached by combining this coupling with setups that otherwise favor species separation, such as a particular combination of atomic scattering lengths [9, 10] or a separation of the respective confining potentials [11]. In this type of setups we find that the effective attraction due to the optical coupling causes an increase in the thickness of the domain wall where the two components physically overlap. More important is the fact that, if we add rotation to a Josephson coupled condensate with separate domains, atoms can use the domain wall to mutate their internal state and shift between components in a continous way. Combining this persistent current in the inner space with a persistent current in real space, the double condensate may now easily create a vortex core for each component in the region where that component has a low density. Even for otherwise small values of the Josephson coupling, these split vortices support a net mass flow comparable to that of conventional vortices. The forma- tion of these novel structures is less costly in terms of angular speed because the vortex of a given component is formed not within its own domain but in the opposite one, where its superfluid density is low and the cost in kinetic energy is therefore small.

To study this type of vortex structure, we analyze first a rotating two-component condensate without optical coupling (i.e. with impenetrable domain walls) and show that its behavior is essentially that of a one-component system with a displaced axis of rotation. Then we show that vortex formation is strongly inhibited because of the ability of the system to gain angular momentum by merely distancing itself from the rotation axis. The picture changes qualitatively when a Raman coupling is introduced to permit coherent hopping between the two internal states. Because the flow of particles in a given state is no longer a conserved quantity, the circulation lines of a component can cross the domain wall with a concomitant decrease in their supporting superfluid density. This results in a global structure of two asymmetrical vortices where matter is efficiently transported both in real space and within the hyperfine doublet.

The model.- In this paper we focus on double condensate systems such as those made of Rb in JILA [1], but this time in rotating traps and with a permanent optical coupling between the species. In the rotating frame of reference [5] the Gross-Pitaevskii equations for the condensate wavefunctions read

$$
\begin{aligned}
i \hbar \partial_{\tau} \Psi_{1} & =\left[H_{1}+\sum_{j} U_{1 j}\left|\Psi_{j}\right|^{2}\right] \Psi_{1}-\frac{1}{2} \hbar \Omega_{R} \Psi_{2}, \\
i \hbar \partial_{\tau} \Psi_{2} & =\left[H_{2}+\sum_{j} U_{2 j}\left|\Psi_{j}\right|^{2}\right] \Psi_{2}-\frac{1}{2} \hbar \Omega_{R} \Psi_{1} .
\end{aligned}
$$

Here $H_{j}$ correspond to the non-interacting Hamiltonians $H_{1,2}=-\frac{\hbar^{2}}{2 m} \Delta+V\left(\mathbf{r}-\mathbf{r}_{1,2}\right)-\hbar \Omega L_{z}+\hbar \bar{\delta}_{1,2}$, where $V(\mathbf{r})=\frac{1}{2} m \omega^{2} r^{2}$ is the trapping potential, $\mathbf{r}_{j}$ is the center of the trap for component $j$, and $\Omega$ is the angular speed. The mutual interactions $U_{i j}=4 \pi \hbar^{2} a_{i j} / m$, are proportional to the $s$-wave scattering lengths $a_{i j}$. The 

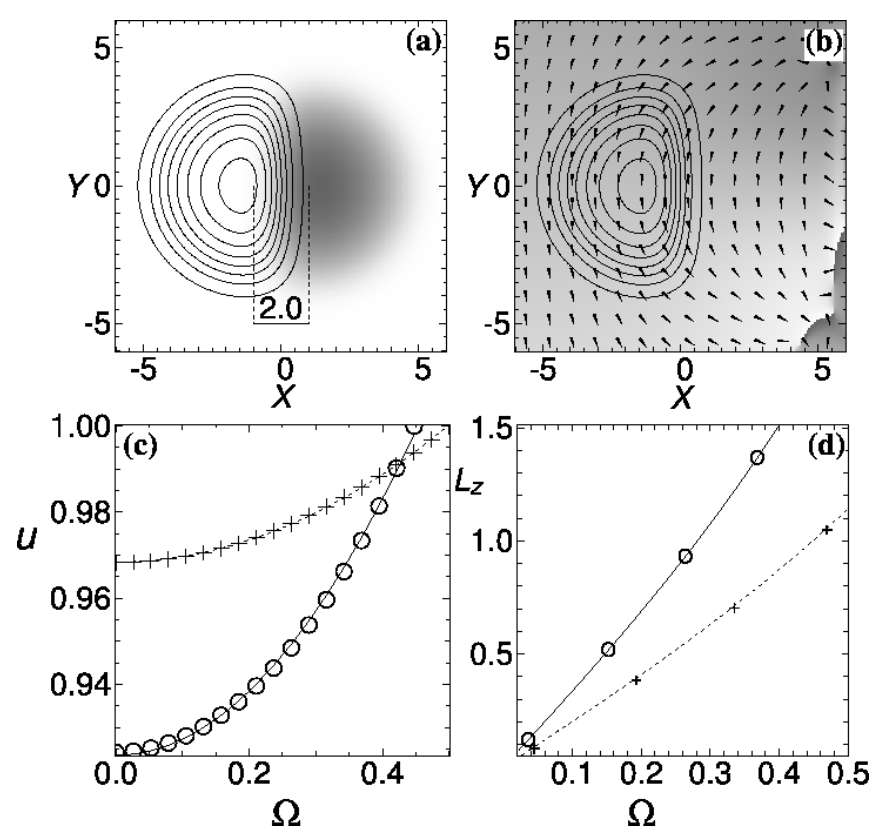

FIG. 1: (a) Density profiles in scenario A for $\Omega=0$. We show a grayscale plot of $\left|\psi_{1}\right|^{2}$, (dark), and a contour plot of $\left|\psi_{2}\right|^{2}$, (solid lines). The trap separation is indicated with dashed lines. (b) Type A condensates for $\Omega=0.135$. Grayscale plot for the phase $\left(\theta=\arg \psi_{1}\right)$, arrows for the direction of the velocity field $(\mathbf{v}=\nabla \theta)$, and contour lines for $\left|\psi_{1}\right|^{2}$. (c) Separation of the atomic clouds and (d) angular momentum as a function of the angular speed for scenarios A (circles, solid line) and B (crosses, dashed line). All magnitudes have been adimensionalized.

last term in each equation models an optical coupling between species. This is a Josephson-type coupling that allows atoms to change their internal state. Since we focus on stationary configurations, the normalization of each wavefunction is fixed, $N_{i}=\int\left|\Psi_{i}(\mathbf{r})\right|^{2} d \mathbf{r}$. Finally, $\hbar \bar{\delta}_{i}$ is a tunable energy splitting in the hyperfine space, which controls the population of each component.

In this paper we restrict our attention to axially symmetric two-dimensional configurations which may describe pancake type traps [12]. In such traps the nonlinear parameter is affected by a corrective factor [5, 13] and $N_{1}$ and $N_{2}$ are interpreted as effective number of particles. To ease the analysis we introduce dimensionless variables $\mathbf{x}=\mathbf{r} / a_{0}$ and $t=\tau / T$. With this change and $\psi_{j}(\mathbf{x}, t)=N_{j}^{-1 / 2} \Psi_{j}(\mathbf{r}, \tau)$, we write the equations for stationary states

$$
\begin{aligned}
& \mu_{1} \psi_{1}=\left[\mathcal{H}_{1}+\sum_{j} g_{1 j}\left|\psi_{j}\right|^{2}\right] \psi_{1}-\lambda \psi_{2}, \\
& \mu_{2} \psi_{2}=\left[\mathcal{H}_{2}+\sum_{j} g_{2 j}\left|\psi_{j}\right|^{2}\right] \psi_{2}-\lambda \psi_{1},
\end{aligned}
$$

with rescaled Hamiltonians

$$
\mathcal{H}_{1,2}=\frac{1}{2}\left[-\Delta+\left(x-x_{1,2}\right)^{2}+y^{2}\right]+i \Omega\left(x \partial_{y}-y \partial_{x}\right) .
$$

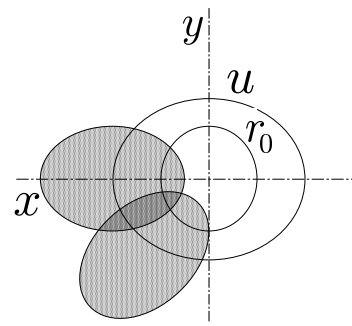

FIG. 2: Scheme of an off-axis rotating trap. Both the center of the trap and the trap itself rotate with angular speed $\Omega$.

In Eq. (2) the splittings $\bar{\delta}_{i}$ are included in the chemical potentials. The parameter $\lambda=2 \Omega_{R} / \omega$ measures the intensity of the optical coupling. Although it is widely tunable, in our work it will be spatially uniform and at most of order unity. We have solved numerically Eq. (2), looking for the solutions that have lower energy, the so called ground states. Each of such solutions represent a stable, experimentally realizable configuration.

We will consider two different scenarios in which the double condensate exhibits domain walls, and which give qualitatively similar results. The first case, which we call "setup B", corresponds to a situation in which $g_{11}=$ $g_{22}=N, g_{12}=2 N$, with a choice of $N=38$. In this case the inequality $g_{12}^{2}>g_{11} g_{22}$ is satisfied and the domains form spontaneously [10] with no need for trap separation nor splitting, i. e. $x_{1,2}=0, \mu_{1}=\mu_{2}$.

The second and most important scenario, which we call "setup A" corresponds to the case of ${ }^{87} \mathrm{Rb}$ [1] with parameter values $\left(\begin{array}{ll}g_{11} & g_{12} \\ g_{21} & g_{22}\end{array}\right)=\left(\begin{array}{cc}1 & 0.94 \\ 0.94 & 0.97\end{array}\right) \times \alpha N$ (i.e. $N_{1}=$ $N_{2}=N$ ), and a typical effective value of $N=100$. This type of condensates has been studied in many previous experimental and theoretical works. For ${ }^{87} \mathrm{Rb}$, the inequality mentioned above is close to saturation [10] and a separation $x_{1}=-x_{2}=1$ ensures the formation of two different domains. The external splitting takes small values, $\mu_{1}-\mu_{2} \leq 0.01$, and it does not influence the results.

Rotation without Josephson coupling.- Here we consider the case $\lambda=0$. The existence of domain walls implies that the motion of species is spatially constrained. Therefore, if we impose some angular speed to the traps containing the condensates, each cloud tends to slip tangentially to the domain wall. The density distribution is similar to the case without rotation, but now, due to the centrifugal force, the species separate a little and gain linear speed. Together with the deformation of the clouds, this mechanism permits the acquisition of a large amount of angular momentum without generating vortices [Fig. [1(d)], which only form at very high $\Omega$.

In Fig. 1 (a) we show the ground state of the rotating double condensate system for $\lambda=0$, showing a structure of domain walls that persists in the presence of rotation and prevents the formation of vortices, as revealed by the phase and circulation patterns of Fig. 1(b). From Fig. 1 (c) it is evident that the both the mutual repulsion and 
the rotation of the trap increase the separation among species. The flow pattern presents a curvature which is too small [Fig. 11(b)], and the gain of angular momentum [Fig. 11(d)] is due instead to the displacement of the condensate away from the origin.

To get a deeper understanding of this configuration it is useful to study the off-axis rotation of a single component condensate. It experiences a potential $V(\mathbf{x}, t)=\frac{1}{2}(\mathbf{x}-$ $\left.\mathbf{r}_{0}(t)\right) A(t)\left(\mathbf{x}-\mathbf{r}_{0}(t)\right)$ with $A(t)$ and $\mathbf{r}_{0}(t)$ rotating at speed $\Omega$ around a displaced axis, as depicted in Fig. 2. Thanks to a particular symmetry of the nonlinear Schrödinger equation with harmonic potential 14, any solution can be written as

$$
\psi(\mathbf{x}, t)=\phi(\mathbf{x}-\mathbf{u}, t) e^{i m \dot{\mathbf{u}} \cdot \mathbf{x} / \hbar+f(t)}
$$

where $\phi(\mathbf{x}-\mathbf{u})$ is centered on the center of mass $\mathbf{u}$ and satisfies the Gross-Pitaevskii equation (2) for $\mathbf{r}_{0}=0$. In the case of radially symmetric traps, $A=\omega^{2}$, we find $\left(1-\Omega^{2} / \omega^{2}\right) \mathbf{u}=\mathbf{r}_{0}$. This tells us that the behavior of a condensate in an off-axis rotating trap is qualitatively similar to that of a condensate in a centered trap: Vortices nucleate at similar angular speeds and they all appear inside the condensed cloud. The difference is that now the condensate has an additional source of angular momentum due to its displacement with respect to the origin, $L_{z} \propto \Omega|u|^{2}$.

These arguments, which are rigorous for the single condensate system, may be extended to the two-component condensate case where the overlap between clouds is small, meaning that vortices should appear in the center of the corresponding clouds even when the trap centers are displaced with respect to the axis of rotation.

Role of Josephson coupling.- In order to explain the role of Josephson coupling let us first consider situation A. It is easy to show that the Josephson terms favor energetically the mixing of both species. In the limit of strong coupling, they coexist in space even when their traps are separated. This is most intuitively appreciated by inspecting the energy functional associated to Eq. (2)

$$
\begin{aligned}
E\left[\psi_{1}, \psi_{2}\right] & =\int\left[\bar{\psi}_{1} \mathcal{H}_{1} \psi_{1}+\bar{\psi}_{2} \mathcal{H}_{2} \psi_{2}\right] \\
& +\int\left[\sum_{i, j=1,2} g_{i j}\left|\psi_{j}\right|^{2}\left|\psi_{i}\right|^{2}-\lambda \operatorname{Re}\left(\bar{\psi}_{1} \psi_{2}\right)\right](5
\end{aligned}
$$

The coupling term by itself is minimized with a solution such that $\arg \psi_{1}=\arg \psi_{2}$. The analogy with the off-axis rotation of a single condensate [see Eq. (何] suggests the variational wavefunction

$$
\begin{aligned}
& \psi_{1}(x, y) \propto e^{-(x-u)^{2} / 2-y^{2} / 2} e^{i v y} \\
& \psi_{2}(x, y) \propto e^{-(x+u)^{2} / 2-y^{2} / 2} e^{-i v y}
\end{aligned}
$$

Substituting this ansatz into Eq. (5) one gets an effective
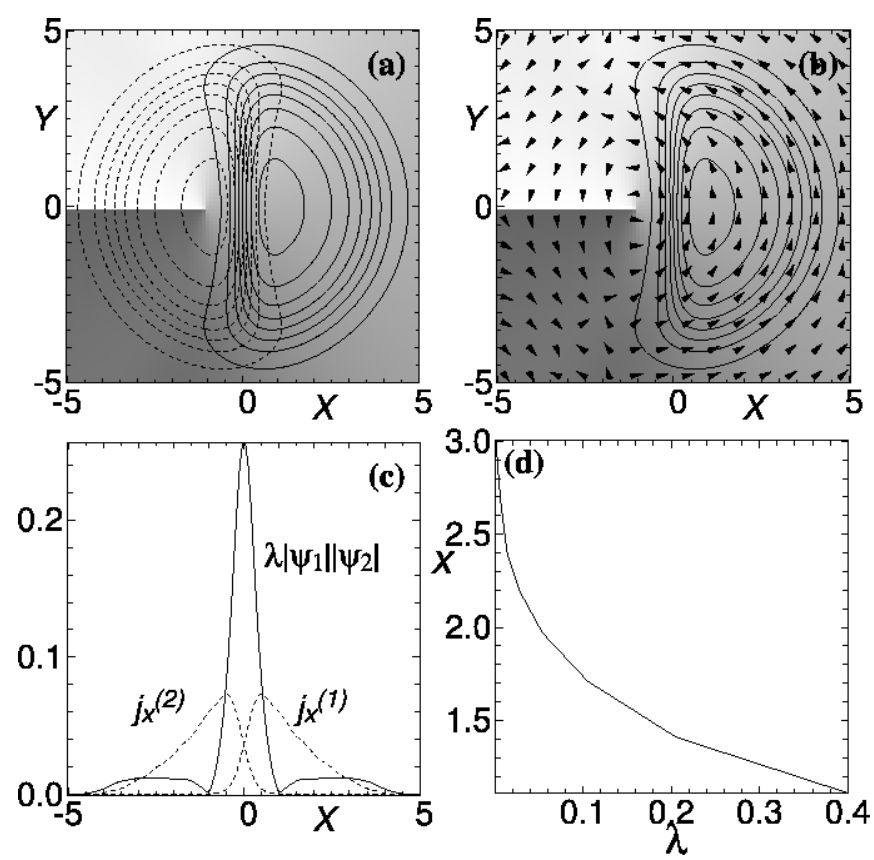

FIG. 3: Rotating two-component condensate with coupling $\lambda=0.1$, scenario B with $N=100$ and angular speed $\Omega=0.2$. (a) Contour lines for the moduli of the wavefunctions $\left|\psi_{1}\right|$ (solid line) and $\left|\psi_{2}\right|$ (dashed line). (b) Contour levels for $\left|\psi_{1}\right|$, phase $\left(\theta=\arg \psi_{1}\right.$, grayscale), and velocity field $\left(\mathbf{v}_{1}=\right.$ $\nabla \arg \psi_{1}$, triangles). (c) Condensate fluxes $j_{x}^{(i)}=\left|\psi_{i}\right|^{2} v_{x}^{(i)}$, together with intensity of the optical coupling, $\lambda\left|\psi_{1}\right| \psi_{2} \mid$, along the line $y=-0.097$. (d) Position of the vortex for a type B condensate with $\Omega=0.28$, as a function of the coupling. All units have been adimensionalized.

energy

$$
E \sim \frac{1}{2}(u-d)^{2}+\frac{1}{2} v^{2}+\Omega u v-\lambda e^{-u^{2}}+g_{12} C e^{-2 u^{2}},
$$

where $C$ is of order unity. Minimization with respect to the velocity $v$ leads to $v=-\Omega u$, and thus $\Omega$ favors separation. More importantly, it is clear from (7) that $\lambda$ effectively decreases the repulsion between condensates. Thus, the separation $u$ decreases with the strength of the optical coupling and becomes zero for a strong enough value of the Josephson coupling, $\lambda_{c}$. Both the repulsive interaction among bosons and the rotation of the trap, tend to inhibit mixture of species, thus increasing the value of $\lambda_{c}$.

Vortex formation.- The structure of matter flow in the condensate suffers a drastic trasformation when the Josepson coupling is introduced. From numerical solutions of Eqs. (2) for setups A and B, we see that any nonzero coupling allows the formation of vortices [Figs. 3(a) and 4 . These vortices involve a matter flow which is orthogonal to the wall separating both condensates [Fig. 3(b)]. We note that the exact location of both vortex cores depends on the intensity of the coupling [Fig. 3(d)].

The mathematical reason for this striking change is that, with a finite value of $\lambda$, the matter flow of each 

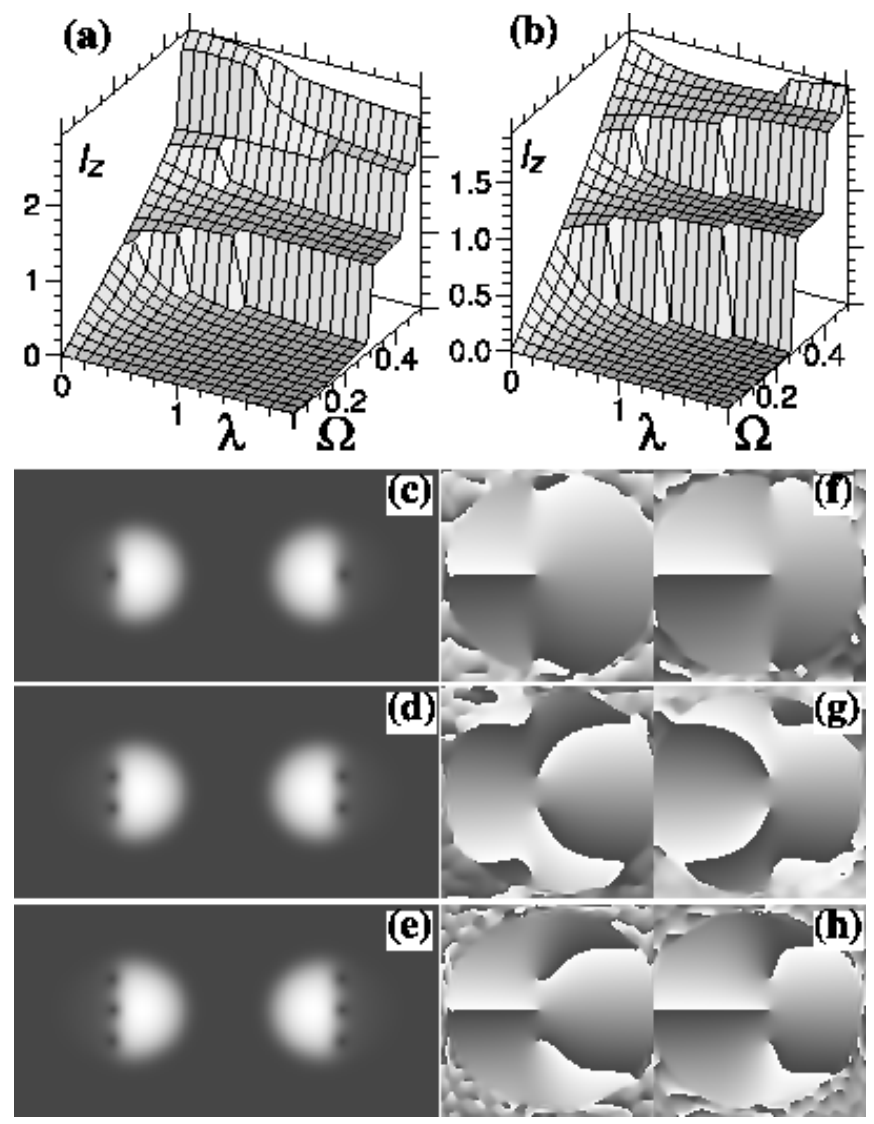

FIG. 4: (a-b) Angular momentum per particle, $l_{z}$, versus $\Omega$ and $\lambda$. (a) type A condensates (b) type B condensates. (c-e) Density plots and ( $\mathrm{f}-\mathrm{h}$ ) phase plots of states of a type A rotating condensate: $\lambda=0.315, \Omega=0.316(\mathrm{c}, \mathrm{f}), \Omega=0.447(\mathrm{~d}, \mathrm{~g})$ and $\Omega=0.474(\mathrm{e}, \mathrm{h})$. In (c-h) we plot both species separately (although they overlap). All magnitudes are dimensionless.

species is no longer conserved. Assuming a divergence free flow, the equation of continuity along the trajectory of a boson becomes

$$
\frac{\partial\left|\psi_{1}\right|^{2}}{\partial l} v_{1}=-\lambda \operatorname{Re}\left(\bar{\psi}_{1} \psi_{2}\right)=-\frac{\partial\left|\psi_{2}\right|^{2}}{\partial l} v_{2}
$$

Thus, as the current line is closed around the origin, there is an exchange of bosons among components. A typical boson flowing around the origin suffers a transformation from state $|1\rangle$ to $|2\rangle$ and viceversa as it completes a circle.

Physically, there is a fundamental reason why bosons are transferred from one component to the other. For $\lambda=0$, vortices must lay inside each condensed cloud, due to current conservation. However, for any nonzero coupling, vortices may appear outside the bulk of the clouds, at a variable distance from the origin. Placed at low density regions, the twist of the phase requires less kinetic energy $\int\left|\psi_{i}\right|^{2}\left(\nabla \arg \psi_{i}\right)^{2}$ and provides more angular momentum than the mere separation of clouds.

In Figure $1(\mathrm{a}-\mathrm{b})$ we plot the angular momentum per particle as a function of the Josephson coupling and the angular speed. The cliffs on the surface are due to the nucleation of successive vortices. The separation of these vortices from the $x=0$ domain wall decreases very fast with increasing $\lambda$ : Fig. 3(d) shows that, already for $\lambda=$ 0.05 , the vortices become visible. As $\lambda \rightarrow 0^{+}$for fixed $\Omega$, vortices disappear continuously moving their core to infinity. To create vortices at $\lambda=0$, one needs to reach a much higher angular speed, $\Omega_{c}$, comparable to that of single component condensates 顿. The upshot is that the optical coupling permits the formation of split vortices with important mass flow at lower angular velocities.

Internal Josephson dynamics.- The Hamiltonian (5) may be written as that of a nonrigid pendulum [8] with an effective interaction energy $E_{c}=\sum_{i j} g_{i j} \sigma_{i j}(-1)^{i+j}$, where $\sigma_{i j} \equiv \int\left|\psi_{i}\right|^{2}\left|\psi_{j}\right|^{2}$, and an effective Rabi frequency $\omega_{R}=\Omega_{R} s_{12}$, with $s_{12} \equiv \int \bar{\psi}_{1} \psi_{2}$. The conclusion is that, for the setups considered here $(\lambda \lesssim 0.5)$, the internal two-state dynamics lies in the collective Josephson regime $\left(2 \omega_{R} / N \ll E_{c} \ll N \omega_{R} / 2\right)$, while in the JILA experiment [6], where no walls are formed, the same dynamics lies in the noninteracting Rabi limit $\left(E_{c} \ll 2 \omega_{R} / N\right)$.

This work has been partially supported by Ministerio de Ciencia y Tecnología under grants BFM2000-0521 and PB96-0080-C02.

[1] M. R. Matthews, B. P. Anderson, P. C. Haljan, D. S. Hall, C. E. Wieman, E. A. Cornell, Phys. Rev. Lett. 83, 2498 (1999).

[2] K. W. Madison, F. Chevy, W. Wohlleben, J. Dalibard, Phys. Rev. Lett. 84, 806 (2000); F. Chevy, K. W. Madison, J. Dalibard, Phys. Rev. Lett. 85, 2223 (2000); J. R. Abo-Shaeer, C. Raman, J. M. Vogels, and W. Ketterle, Science 292, 476 (2001).

[3] See e.g. A. Svidzinsky, A. L. Fetter, Jour. Phys. B 13, R135 (2001), and references therein.

[4] D. L. Feder et al. Phys. Rev. Lett. 86, 564 (2001); F. Dalfovo, S. Stringari, Phys. Rev. A 63, 011601 (2001).

[5] J. J. García-Ripoll and V. M. Pérez-García, Phys. Rev. A 64, 013602 (2001).

[6] D. S. Hall, M. R. Matthews, C. E. Wieman, E. A. Cornell, Phys. Rev. Lett. 81, 1543 (1998).

[7] B. P. Anderson and M. A. Kasevich, Science 282, 1686 (1998).

[8] For a review, see F. Sols, in Proceedings of the International School of Physics "Enrico Fermi", M. Inguscio, S. Stringari, and C. E. Wieman, eds. (IOS Press, Amsterdam, 1999), p. 453.

[9] B. D. Esry, C. H. Greene, Nature 392, 434 (1998).

[10] S. Coen, M. Haelterman, Phys. Rev. Lett. 87, 140401 (2001).

[11] D. S. Hall, M. R. Matthews, J. R. Ensher, C. E. Wieman, E. A. Cornell, Phys. Rev. Lett. 81, 1539 (1998).

[12] A. Gorlitz et al., arXiv: condmat/0104549.

[13] J. J. García-Ripoll, G. Molina-Terriza, V. M. PérezGarcía, and L. Torner, Phys. Rev. Lett. 87, 140403 (2001).

[14] J. J. García-Ripoll, V. M. Pérez-García, and V. Veksler- 
chik, Phys. Rev. E, in press.

[15] M. Linn et al., arXiv: condmat/0103489. 